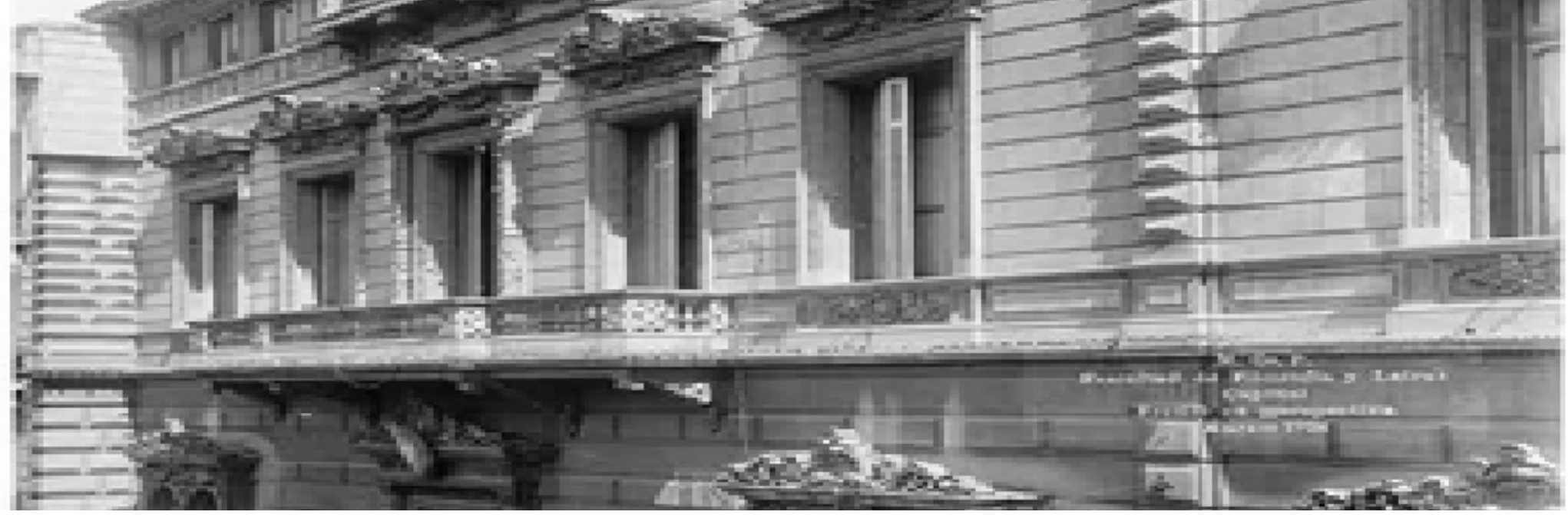

\title{
History of the German philology in Argentina (Chapter 7 ) - GESCHICHTE DER ARGENTINISCHEN GERMANISTIK
}

\begin{tabular}{|c|c|}
\hline Authors: & Lila Bujaldon de Esteves \\
\hline Submitted: & 16. February 2019 \\
\hline Published: & 19. February 2019 \\
\hline Volume: & 6 \\
\hline Issue: & 2 \\
\hline Affiliation: & $\begin{array}{l}\text { CONICET (Nationaler Rat für wissenschaftliche und } \\
\text { technologische Forschung), UNCUYO (Universidad Nacional de } \\
\text { Cuyo) }\end{array}$ \\
\hline Languages: & German \\
\hline Keywords: & $\begin{array}{l}\text { History, Authors, Literature, Argentina, Germany, Günther Ballin, } \\
\text { Werner Bock, Ilse Brugger. }\end{array}$ \\
\hline Categories: & $\begin{array}{l}\text { DEMETRIOS Literary Works, Humanities, Social Sciences and } \\
\text { Law }\end{array}$ \\
\hline DOI: & 10.17160/josha.6.2.537 \\
\hline
\end{tabular}

\section{Abstract:}

This study of the history of German philology in Argentina describes and analyses the period of time between the Revolution of May 1810, which means after the independence of Argentina from Spain, and the 1950s. The study has its focus on the history of the degree program of the University of Buenos Aires. We are going to read about the first professors of German literature at the University in chapter four, five and six. This last chapter is about Exil und Germanistik in Argentinien, more specific about German and Spanish exiled scholars of the thirties at Argentine universities. Life and work of the exiled Germanist Günther Ballin (1909-1982), Werner Bock (1893-1962) and Ilse M. de Brugger (1908-1995).

\section{JOSHA Jomana olsemence. Humanities and Arts




\section{GESCHICHTE DER ARGENTINISCHEN GERMANSITIK}

\section{Lila Bujaldon de Esteves}

Affiliation: CONICET (Nationaler Rat für wissenschaftliche und technologische Forschung), UNCUYO (Universidad Nacional de Cuyo)

\section{Kapitel VII}

Exil und Germanistik in Argentinien. Deutsche und spanische exilierte Gelehrte der Dreissiger Jahre an argentinischen Universitäten. Leben und Werk des Exilgermanisten Günther Ballin (1909-1982), Werner Bock (1893-1962) und Ilse M. de Brugger (1908-1995).

Die Wissenschaftsgeschichte der Germanistik hat sich in den letzten Jahren des 20. Jahrhunderts für jene Germanisten, die in der Zeit des Nationalsozialismus ins Exil gingen, interessiert. Diese Forschungen ergeben, dass als Folge der akademischen germanistischen Tätigkeit im Ausland eine komparatistische Perspektive entstand, die darauf beruhte, dass das fremde kuturelle Milieu mitgedacht wurde. Die exilierten Germanisten sind über ihr Bild des jeweiligen Aufnahmelandes, in dem sich entfalten mussten, befragt worden. Da sie sich als Vertreter des so genannten "wahren" Deutschland ansahen, übernahmen sie die Lehre der Germanistik als eine Art hohe Aufgabe und betrachteten ihre Arbeit als Widerstand gegen die damalige Korruption der humanistischen Werte in ihrer Heimat. Auch interessierten sie sich sowohl für den Beitrag der jüdischen Schriftssteller zur deutschen Literatur, wie für das Werk zeitgenössischer aus Deutschland exilierter Autoren; sie versuchten, eine entsprechende Methodologie zu entwickeln. In Argentinien finden wir unter diesen Germanisten Werner Bock, Ilse M. de Brugger und Günther Ballin, deren Weg ins Exil und deren akademische Laufbahn im Ausland in diesem Kapitel behandelt werden. Da es bislang an

Argentum virtus robur et studium - La virtud argentina es la fuerza y el estudio Die argentinische Tugend ist die Kraft und das Studium (das Studieren) 
genauen biographischen Daten über sie und andere nach Argentinien ausgewanderte Akademiker mangelt, fehlt in den vorliegenden im Exil entstandenen Ausgaben und in den entsprechenden Untersuchungen eine vollständige namentliche Erfassung dieser Germanisten. Dieses Kapitel verscuht dem Mangel abzuhelfen. Auch soll es dazu beitragen, zwischen den Germanisten zu unterscheiden, die in Argentinien Zuflucht fanden und jenen, die ohne den Zwang des Exils in diesen Jahren nach Argentinien kamen.

In den 30er Jahren des XX. Jahrunderts war die argentinische Universität mit dem Prozess ihrer akademischen Spezialisierung beschäftigt. Dazu sollte neben den schon bestehenden Lehrstühlen die Gründung von Forschungsinstituten dienen. Auch unterstützte man nachdrücklich die Einführung neuer Disziplinen und Methodologien, die meist aus Europa stammten. Deswegen suchte die Universtiät ausländische Akademiker zu gewinnen, die helfen sollten, diese Pläne auszuführen. Vor allem führten ab 1939 die spanische Bürgerkriegs und-nachkriegszeit, und im geringerem Masse ab 1933 das deutsche Exil zu einer europäischen Diaspora an den Hochschulen. So erklärt sich das hohe Niveau mancher Disziplinen und Institute an den argentinischen Universitäten in dieser Epoche. Für die spanischen Professoren war es viel leichter als für die Deutschen, sich im akademischen Leben Argentiniens zu integrieren. Dazu trugen die gemeinsame Sprache und die schon bestehende Kontakte mit argentinischen Kollegen und Institutionen bei. Im Gegensatz zu ihnen benötigen mehrere der deutschen Professoren Jahrzehnte zur Anerkennung ihrer mitgebrachten Zeugnisse. Indirekt wurden übrigens viele der neu angekommenen spanischen Hochschullehrer zu Vermittler der deutschen Wissenschaft, weil sie in Deutschland studiert hatte bzw. In Deutschland ausgebildet waren. Auf dem Gebiet der Pädagogik, der Jura oder der Mathematik übersetzten sie deutsche Autoren, deren Werke an den argentinischen Universitäten jahrelang als Lehrbücher verwendet wurden. Ohne den wetvollen Beitrag des Philosophen José Ortega y Gasset zu vergessen, setzten die spanischen Hochschullehrer in den 40Jahren ihre Rolle als Vermittler der deutschen Kultur, insbesondere der deutschen Wissenschaft fort.

Argentum virtus robur et studium - La virtud argentina es la fuerza y el estudio Die argentinische Tugend ist die Kraft und das Studium (das Studieren) 
Für die Deutschen war Argentinien ein Exilland "zweiter Wahl”. Trotzdem nahm von ganz Lateinamerika während der Herrschaft Hitlers Argentinien die höchste Anzahl deutscher Auswanderer auf. Auf Grund der vielen ilegalen Einwanderer variiren die Zahlen zwischen 35.000 und 45.000 deutschen Emigranten. Es handelte sich um eine "qualifizierte" Emigration von Künstlern, Musikern, Ingenieuren, Wissenschaflern, Schauspielern, Unternehmern, Kaufleuten, die das Profil der heutigen Metropole von Buenos Aires mitgeprägt haben. Die meisten von ihnen waren "rassisch" verfolgt. Zu diesen zählen auch die drei erwähnten Germanisten, die in der Zeit des Exils nach Buenos Aires kamen.

\section{$\underline{\text { Über deutsches Exil }}$}

Biographisches Handbuch der deutschsprachigen Emigration nach 1933. Politik. Wirtschaft. Öffentliches Leben. München: Sauer, 1980.

Bujaldon de Esteves, Lila. "Exil und Germanistik in Argentinien”. Peter Wiesinger/Hans Derkits (Hrg). Akten des X. Internationalen Germanistenkogresses Wien 2000.Bd. 11. Bern: Peter Lang, 2003, 285-290.

"Exil und Vermittlung deutscher Kultur. Der Fall der spanischen exilierten Gelehrten in den argentinischen Universitäten ab 1939”. Jahrbuch für Internationale Germanistik, Reihe A, Bd.82, 2007, 87-94.

----------- "Bibliotecas perdidas, bibliotecas recuperadas. Peripecias de las bibliotecas germanísticas argentinas”. Cuadernos del Archivo (print), 1, 2016, 27-46.

Handbuch der deutschsprachigen Emigration 1933-1945. Krohn, Patrick, C.D., von zur Mühlen, G. P. Lutz Wincker (Hrg). Primus Verlag, 1998.

Modernisierung oder Überfremdung? Zur Wirkung deutscher Exilanten in der Germanistik der Aufnahmeländer. Schmitz, W. (Hrg). Stuttgart: Metzler, 1994.

Argentum virtus robur et studium - La virtud argentina es la fuerza y el estudio Die argentinische Tugend ist die Kraft und das Studium (das Studieren) 
Schwartzstein, Dora. Entre Franco y Perón. Memoria e identidad del exilio republicano español en la Argentina. Barcelona: Crítica, 2001.

Wissenschaftsgeschichte der Germanistik in Porträts. Christoph König, Hans-Harald Müller und Werner Röcke (Hrg). Berlin: de Gryter, 2000.

\section{$\underline{\text { Günther Ballin (1908-1982) }}$}

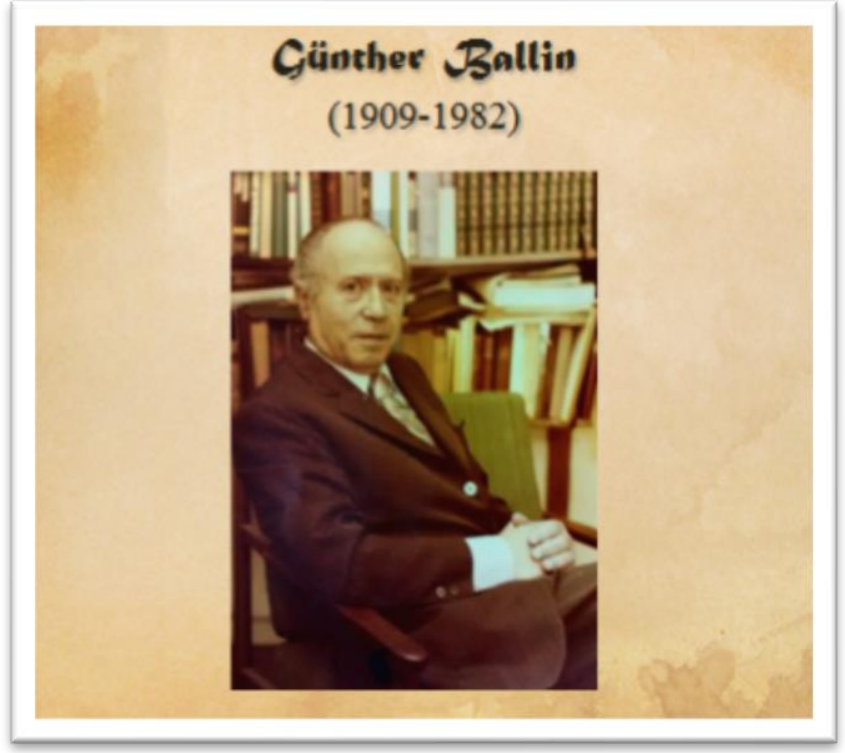

Günther Ballin ist 1938 mi einem Touristenvisum in Buenos Aires angekommen. Vor seiner akademischen Einstellung hatte Ballin oft Gedichte und Erzählungen im Argentinischen Tageblatt und in der Jüdischen Wochenschau veröffentlicht. Er war Kanzelredner in vielen jüdischen Gemeinden der argentinischen Hauptstadt bekannt und als Lehrer an der Pestalozzi-Schule tätig, die 1934 von politisch und rassistisch verfolgten Emigranten gegründet worden war. Sein besonderes Interesse an der Problematik der Jugendlichen ist auf die Zeit seiner Mitarbeit im Schwarzen Fählein zurückzuführen. Mit seinen Aktivitäten versuchte das Schwarze Fähnlein die deutsche Identität seiner assimilierten jüdischen Mitglieder zu stärken. 1934 wurde das Schwarze Fähnlein von der Regierung aufgelöst, da diese "Judenschaft" ein den Projektionen der Nazis entgegen gesetztes Bild von den Juden vermittelte. Günther Ballin hatte das Bismarck -Gymnasium in Berlin besucht. Zwischen 1927 und 1932 studierte er Geschichte und Germanistik an der Friedrich-Wilhelm Universität seiner Heimatstadt. 1932 nach seiner Promotion in Berlin, nahm Ballin das Studium an der Lehranstalt für die 
Wissenschaft des Judentums wieder auf. Er fiktionalisierte die Wendung seiner eigenen Identität durch die Entdeckung der jüdischen Wurzeln des Protagonisten in seinem 1945 im Exilverlag Cosmopolita erchienenen autobiographischen Roman “Zwischen Gestern und Morgen”. In seinen Memoiren erzählt Günther Ballin von seiner Berliner Kindheit und der jüdisch-sozialistischen Umbegung ; auch zeichnet er kritische Portraits seiner damaligen Dozenten und Professoren. Als Germanist interessierte er sich für die im Exil entstandenen Romane Lion Feuchtwangers, Erich Maria Remarques, H. Kestens und Thomas Manns. Günther Ballin hinterliess auch wichtige Abhandlungen über die Geschichte der deutschsprachigen Juden in Argentinien. 1955, am Ende der peronistischen Ära, als sich Juan C Probst aus der Universität zurückzog, konnte Günther Ballin bis zum Jahr 1965 den Lehrstuhl für Deutsche Literatur der Universität Buenos Aires übernehmen.

\section{Ausgewählte Bibliographie}

Belletristisches und Autobiographie

Zwischen Gestern und Morgen (Roman). Buenos Aires: Cosmopolita, 1945.

Erinnerungen. Es war je erst gestern. 2Bde. Buenos Aires 1979-1980.

Publikationsorgane für zahlreiche Aufsätze

Berliner Zeitung am Mittag (1929-1933). Argentinisches Tageblatt (1942-1952). Jüdische Wochenschau (1944-1956). La Prensa (1964-1968). Aufbau, New York (1972).

Über Günther Ballin

Bujaldón de Esteves, Lila. "Wird die Fremde dritte Heimat? Problema deutsch-jüdischer Exilschriftsteller in Argentinien (über G.B.,W. Bock und Hans Silber)". Akten des VIII. Internationalen Germanisten-Kongressses. Joshinori Shichiji (Hrg). Bd. 8. Tokyo 1990, 284-291. 


\section{$\underline{\text { Werner Bock (1893-1962) }}$}

Bei Werner Bock liegt eine germanistische Tätigkeit vor, die sich ausserhalb der Universität abspielte. Durch selbstständige Publikationen und zahlreiche Beiträge in den wichtigsten Zeitschriften und Zeitungen der 40er und 50er Jahren vermittelte Bock die Kenntnis deutscher Autoren, insbesondere von Goethe, von den Romantikern, Stefan George, Karl Wolfskehl und Hermann Hesse. 1949

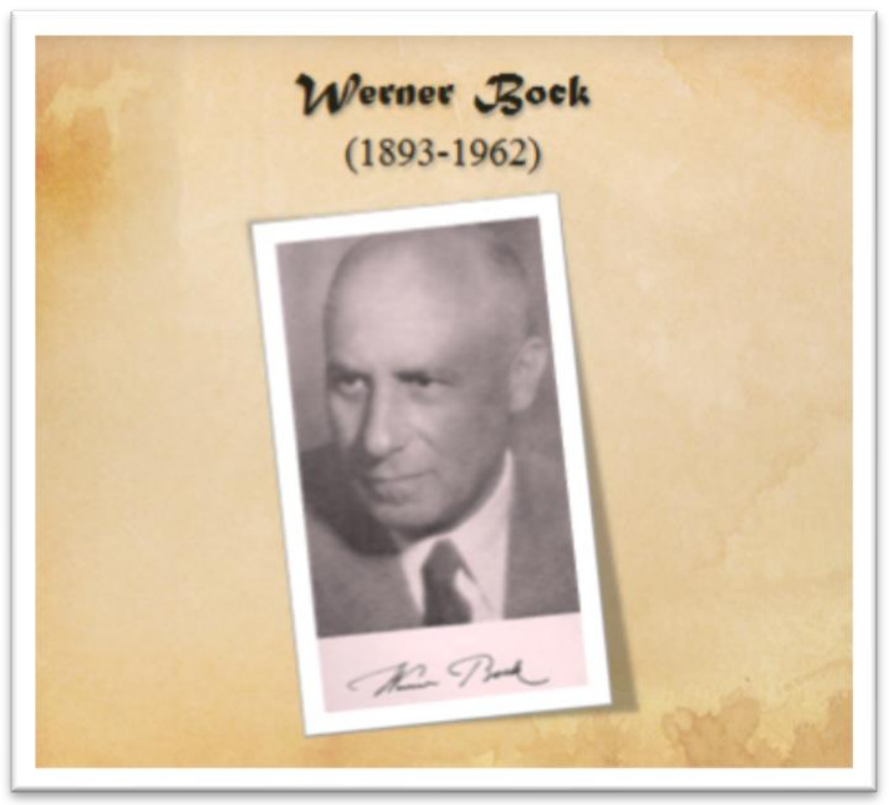
veranstaltete er Goethe-Feiern, die ein breites deutsches und lokales Publikum anzogen. Zwischen 1946 und 1949 gelang es ihm, in Montevideo Deutsche Philosophie und Literatur zu lehren. Bock stammte aus einer jüdischen assimilierten Bürgerfamilie, er hatte in Giessen über Martin Wieland promoviert. Bis zu seiner Emigration 1939 konnte er sich seinem Traumberuf als Schriftsteller nicht widmen. Mit Hilfe von Empfehlungsbirefen des Philosophen Hermann von Keyserling fand er schnell in den führenden europäisch orientierten kulturellen Kreises Argentiniens einen Platz. Zusammen mit dem Exildichter Paul Zech war Bock in Argentinien verantwortlich für die Herausgabe der wichtigsten Exilpublikation Südamerikas, der "Deutschen Blätter" (Santiago de Chile). 1958 siedelte Bock in die Schweiz um, wo er sich der Verbreitung der argentinsichen Literatur, der Herausgabe der väterlichen Tagebücher und dem eigenen dichterischen Schaffen widmete.

Argentum virtus robur et studium - La virtud argentina es la fuerza y el estudio Die argentinische Tugend ist die Kraft und das Studium (das Studieren) 
$\underline{\text { Bellestrisches und Essays (Auswahl) }}$

Das ewige Du (Gedichte), Weimar, 1931. El eterno Tú (Erzählungen). Buenos Aires, 1943. Morir es nacer (Erzählungen). Buenos Aires, 1947. Tröstung. Auswahl der Gedichte aus den Jahren 1909-1950. Buenos Aires, 1951. Blüte am Abgrund. Prosaauswahl meiner Schaffensjahre 19191950. Buenos Aires, 1951.

Idea y amor: de Goethe a Hesse. Buenos Aires, 1952. Momento y eternidad. Ensayos sobre literatura alemana. Buenos Aires, 1957.

Publikationsorgane für zahlreiche Aufsätze (Auswahl)

Argentinisches Tageblatt (1940-1954), La Nación (1943-1958), Deutsche Blätter/Hojas Alemanas (1944-1946), Sur (1951-1956), Südamerika (1951-1957), Merkur (1953-1962), Universitas (19541960), Akzente (1958-1961), Humboldt (1960-1962), u.a.

Über Werner Bock

Bujaldon de Esteves, Lila. "El tema del exilio en la obra literaria de Werner Bock". Humboldt $71 / 21,1980,53-65$.

Latein Amerika and the Literature of Exile. Moeller, Hans-B. (Hrsg) Heidelberg: Universitätsverlag Winter, 1983.

Unseld, Kerstin. Werner Bock- Ein deutscher Literat am Rio de la Plata. Heildelberg: Universitätsverlag Winter, 2009. 


\section{Ilse Brugger (geb. Masbach) (1908-1995)}

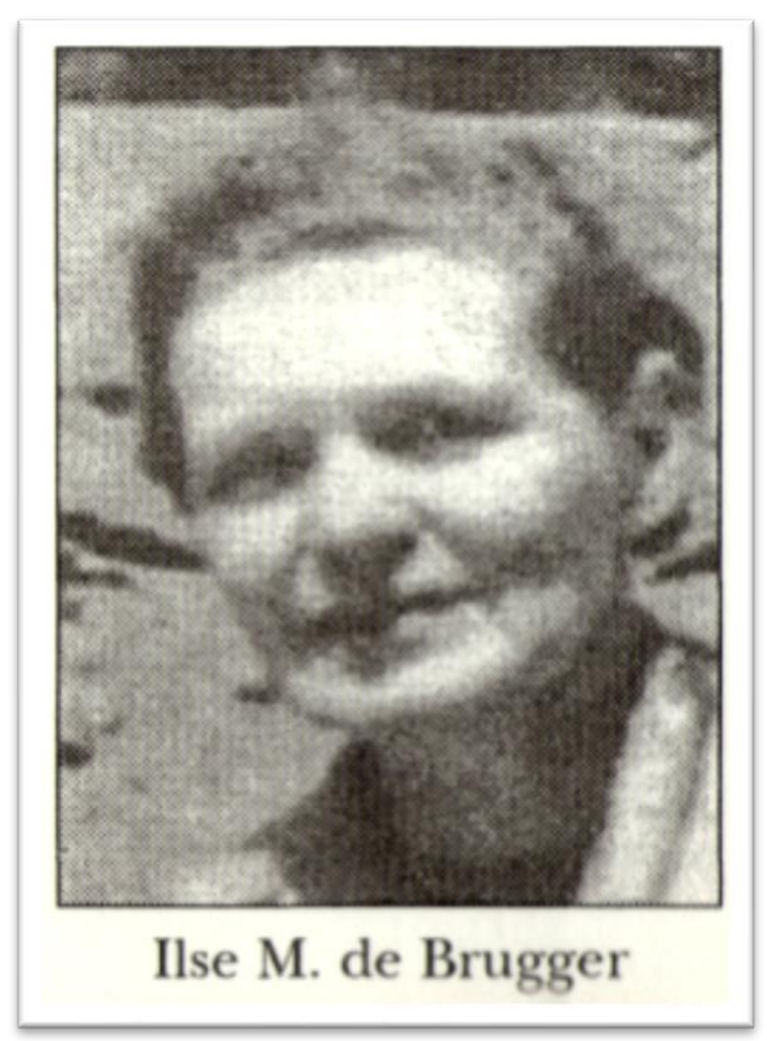

1938 war die dreissigjährige Schriftstellerin und Germanistin Ilse Brugger mit ihrem Ehemann und ihrem jüdischen Vater in Buenos Aires angekommen. Ihr Ausschluss aus der Reichsschrifttumskammer im Jahr 1935 hatte ihre Arbeitschanchen in Deutschland beendet. 1941 gewann das Instituto de Estudios Germánicos der Philosophischen Fakultät der Universität Buenos Aires sie als neue Mitarbeiterin. Sie erlangte die zweite weibliche Professur in der Geschichte dieser Fakultät. Das Datum ihrer ersten Studiensemester in Berlin wie auch das Jahr ihrer Geburt und Promotion erlauben es. Ilse Brugger mit Liselotte Blumenthal, Käthe Hamburger u.a. in die erste Generation der deutschsprachigen Literaturwissenschaftlerinennen einzureihen. Das Todesproblem bei Rainer Maria Rilke war 1930 das Thema ihrer Innsbrucker Promotion. 1944 erschien das Buch auf Spanisch in Buenos Aires und trug zu der frühen und begeisterten Rezeption des Dichters in Argentinien bei.

1957 wurde Brugger die erste Professorin für Deutsche Literatura n der Universität La Plata und war ab 1965 auch an der päpstlich-katholischen Universität Buenos Aires tätig. Ihre auf Spanisch verfassten Bücher über deutsches Theater des Expressionismus, über das deutsche Theater des 20. Jahrhunderts und das Zeitalter Goethes und Schillers gehören noch heute an den argentinischen Universitäten zur empfohlenen germanistischen Sekundärliteratur. Auch ihre Übersetzungen von Dramen Grillparzers, Hebbels und Hugo von Hofmannsthals und von Schillers Wallenstein in Argentum virtus robur et studium - La virtud argentina es la fuerza y el estudio Die argentinische Tugend ist die Kraft und das Studium (das Studieren) 
spanischen Zwölfsilbern verdienen neue Auflagen. In ihren zahlreichen germanistischen Publikationen findet sich seltsamerweise keine Spur von den typischen Merkmalen, die die Exilgermanistik kennzeichnen, was man als Mechanismus der Überadaptation interpretieren kann.

\section{$\underline{\text { Selbständige Publiktionen (Auswahl) }}$}

Teatro alemán expresionista. Buenos Aires: La Mandrágora, 1959.

Teatro alemán del siglo XX. Buenos Aires: Nueva Visión, 1961.

Expresionismo. Buenos Aires: Centro Editor de América Latina, 1968.

El romanticismo alemán. Capítulo Universal n 7. Buenos Aires: Centro Editor de América Latina, 1968.

Publikationsorgane für zahlreiche Aufsätze und Übersetzungen

Estudios Germánicos, Boletín de Estudios Germánicos, La Nación, Revista de la Universidad de La Plata, Südamerika, Hebbel-Jahrbuch, Fascículos de la Antología Alemana.

Über Ilse Brugger

"Ich hatte meinen Rhythmus verloren". Ein Gespräch mit Dr. Ilse Brugger-Masbach. Argentinisches Tageblatt, 14.9.1991.

Bujaldon de Esteves, Lila. "Ilse Brugger (geb. Masbach)". Internationales Germanistenlexikon 1800-1950. Hrg. Christoph König. Berlin: De Gruyter, 2003, 283-284. 